\title{
HEGEL UND DIE GESCHICHTE CHINAS
}

\section{Von Wolfgang Franke}

Im späten 17. und frühen 18. Jahrhundert sahen einige der bedeutendsten europäischen Denker jener Zeit, insbesondere Gottfried Wilhelm von Leibniz, die chinesische Kultur als der abendländischen zumindest ebenbürtig, wenn nicht als überlegen an, und die politisch-sozialen Verhältnisse Chinas schienen ihnen nahezu vorbildlich zu sein. Tatsächlich waren zu jener Zeit, als das traditionelle China seine letzte politische, wirtschaftliche und kulturelle Blütezeit erlebte, die Verhältnisse in China in ziemlich jeder Beziehung günstiger als in dem noch unter den Folgen des Dreißigjährigen Krieges daniederliegenden Europa. Durch eine zuweilen etwas tendenziöse Redaktion der Berichte, die von Jesuiten-Missionaren aus China kamen, mußte sogar der Eindruck entstehen, als herrschten in China vollends ideale $\mathrm{Zu}$ stände.

Doch änderte sich das Chinabild in Europa schnell. Den idealisierenden Berichten der frühen Jesuiten folgten bald die ins entgegengesetzte Extrem verfallenden geringschätzigen Schilderungen der europäischen Händler und Seefahrer und dann auch der christlichen Missionare des 19. Jahrhunderts. Das Interesse und die Hochachtung für die chinesische Kultur, die für die Zeit der Aufklärung charakteristisch gewesen waren, wichen dem Streben nach Handelsgewinnen und nach kolonialer Ausbeutung Chinas. An die Stelle der universalen Weltaufgeschlossenheit eines Leibniz. oder Voltaire trat eine enge, allein auf Europa konzentrierte Auffassung. Zurückgreifend auf das mittelalterlich-christliche Weltbild sah sich Europa im 19. Jahrhundert als den Mittelpunkt der Welt, ja als die Welt schlechthin. Diese europäische Hybris gegenüber den außereuropäischen Völkern und Kulturen beschränkte sich nicht auf Politik und koloniale Expansion, sondern zeigte sich in gleicher Weise im Geistesleben.

Hegel ist ein besonders typischer Exponent dieser geistigen Haltung. In der zweiten Hälfte seiner Philosophie der Weltgeschichte, die mit dem Teil über „Die Orientalische Welt" beginnt, steht an erster Stelle ein ausführlicher Abschnitt von mehr als fünfzig Seiten über China. Gewiß reichte das damals Hegel in europäischen Sprachen über China zur Verfügung stehende Material nicht aus, um einen genauen Einblick in die historische Entwicklung Chinas zu erhalten. So ließ die ihm vorliegende, 1777-1785 von De Mailla in Paris herausgegebene dreizehnbändige Histoire générale de la Chine Hegel zu dem etwas voreiligen Schluß kommen:

„Wir können uns in die Einzelheiten dieser Geschichte weiter nicht einlassen, die, da sie selbst nichts entwickelt, uns in unserer Entwicklung hemmen würde, "1

oder

„Insofern hat China eigentlich keine Geschichte.“2

Das chinesische Original, das De Mailla übersetzt hat, ist nämlich nicht eigentlich ein Geschichtswerk, sondern eher eine Art Handbuch der politischen Staatsethik des Konfuzianismus des 12. Jahrhunderts, dargestellt an Beispielen der Geschichte ${ }^{3}$. Über den Charakter dieses Werkes waren sich weder De Mailla noch Hegel im klaren. Aus weiteren Berichten, die Hegel zur Verfügung standen, hätte er aber

1 G. W. F. Hegel, Sämtliche Werke, Bd. IX, Philosophie der Weltgeschichte, Hrsg. Georg Lasson, Leipzig 1923, S. 283.

2 Ebd. S. 278.

3 Wolfgang Franke, China und das Abendland, Göttingen 1962, S. 118. 
zumindest Hinweise auf den außerordentlichen Umfang des chinesischen historischen Schrifttums entnehmen können. Manche seiner Bemerkungen, wie z. B. die über die Veränderungen der Eigentumsverhältnisse im 3. Jahrhundert vor Christo ${ }^{4}$ zeigen, daß er immerhin von einigen grundlegenden Wandlungen in der chinesischen Geschichte Kenntnis hatte, es aber nicht für notwendig hielt, solche Wandlungen $\mathrm{zu}$ beachten. Hegel gab sich mit dem oberflächlichen Eindruck zufrieden, den die ihm vorliegenden ganz wenigen Übersetzungen und Berichte vermittelten, und er erachtete sie als ausreichend, um zu einem so dezidierten Urteil über die chinesische Geschichte zu kommen. Hegel meinte von den Chinesen,

„daß wir jetzt durchaus über China Bescheid wissen. Wir haben so gründliche Kenntnisse ihrer Literatur und ihres ganzen Lebens wie ihrer Geschichte." 5

Er sah keine Veranlassung $\mathrm{zu}$ einer kritischen Einstellung gegenüber den Quellen seiner Information.

Hegels Darstellung der Geschichte Chinas ist ein Durcheinander von Richtigem und Falschem, Verstandenem und Mißverstandenem. Dabei liebt er es, mit einer für den Europäer des 19. Jahrhunderts typischen, hochmütigen Verachtung exotische Details darzustellen:

„Über die Lage ihres Hauses und Begräbnisplatzes haben die Chinesen die größte Not. Damit gehen sie viele Jahre um; und wenn ein anderer ein Haus baut, so kann es ihm die größten Händel erwecken, daß es im Winkel zu ihrem Hause steht. Bögen werden gezogen, es wird geopfert und gefleht, den Schaden abzuwenden, der durch die Nachbarschaft entsteht ... das gehört unter die Rubrik der Geistlosigkeit der Chinesen. “8 Oder

„Für die Genien, von denen jeder auf seine Weise verehrt wird, sind Skulpturbilder festgesetzt. Es sind scheußliche Götzenbilder, die noch nicht Gegenstand der Kunst sind, weil nichts Geistiges darin sich darstellt. Sie sind daher nur erschreckend, furchtbar, negativ. Der Gegenstand der Kunst ist das Geistige, Göttliche; dies hat sie in ihrem sinnlichen Material darzustellen. Aber wo das Religiöse nicht geistig ist, sondern nur ein Gewaltiges, Schreckliches und Furchtbares, da fällt auch seine Darstellung scheußlich aus."7

Die in diesen Sätzen zum Ausdruck kommende Haltung ihres Autors ist ziemlich ähnlich der der Chinesen zur gleichen Zeit; auch sie sahen mit ähnlichem Selbstgefühl auf die „Barbaren des Westens“ herab. Chinesische Beschreibungen Europas aus dem gleichen Zeitraum der ersten Hälfte des 19. Jahrhunderts sind ähnlich anekdotenhaft wie Hegels zitierte Ausführungen, wenn auch etwas weniger unsachlich. So heißt es z. B. über Wien:

„Der Palast des Königs ist besonders stattlich; der König für seine Person ist aber sparsam. Mit dem Volke verkehrt er wie der Vater mit seinen Kindern, und andere Leute behandelt er freundlich. Das Volk hält beharrlich an der Katholischen Religion fest. Die Lehren von bösen Geistern und von der Geomantik sind ihm besonders verhaßt. Die Leute lieben Trinken, Essen und Musik. Die Frauen sind lustig und hübsch, aber wahren keine Keuschheit. Die Vorstadt von Wien hat 36000 Einwohner. In der dortigen Bibliothek gibt

4 G. W. F. Hegel, Sämtliche Werke, Bd. IX, Philosophie der Weltgeschichte, Hrsg. Georg Lasson, Leipzig 1923, S. 284 .

5 Ebd. S. 280.

6 Ebd. S. 326-327.

7 Ebd. S. 327 
es 70000 Bände. In einer benachbarten Stadt ist ein Heiligtum, wo das Volk Weihrauch abbrennt und Götterbilder verehrt. Von den Priestern betört, stiftet es dafür goldenes und silbernes Gerät."8

Hegel stellt nicht nur dem Europäer fremde und unverständliche Sitten und Gebräuche in dieser Weise absprechend dar, sondern er urteilt auch geringschätzig über abweichende gesellschaftliche und institutionelle Entwicklungen in China:

„Der Mangel des ganzen Prinzips der Chinesen liegt darin, daß bei ihnen das Moralische nicht vom Rechtlichen geschieden ist. Eine vernünftige Verfassung muß das Moralische und Rechtliche einer jeden Sphäre für sich hervorbringen. Die orientalische Eigentümlichkeit aber ist die unmittelbare Vereinigung beider Prinzipien. Sie ist vorhanden in dem sittlichen Zustande und in einem Zustande des Staates, bei dem das Sittliche noch das Regierende ist. In solchen Staaten sind dann die Gesetze teils noch dürftig, teils betreffen sie die Sitten."

Hier erkennt Hegel zwar richtig den grundlegenden Sachverhalt der Vereinigung des moralischen und rechtlichen Prinzips, verwirft ihn aber als minderwertig. Den Chinesen galt die Trennung beider Prinzipien im Abendlande als unverständlich und barbarisch. In überheblicher Weise spricht Hegel ferner den Chinesen das Gefühl der Ehre ab ${ }^{10}$, wobei er nicht erkennt, daß ihr Ehrgefühl ein anderes ist als das der Europäer jener Zeit. Er nennt die Chinesen verworfen, unmoralisch:

„Sie sind höchst diebisch und verschmitzt . . , dafür bekannt zu betrügen, wo sie nur irgend können." 11

Ähnliche Äußerungen finden sich in chinesischen Berichten über Europäer. Es berührt seltsam, wenn ein sonst so differenzierter Gelehrter wie Hegel mit solchen Banalitäten argumentiert.

Offenbar konnte auch Hegel dem Geist seiner Zeit nicht entgehen. Nur was sich aus der Entwicklung der abendländischen Kultur ergab und was irgendwie für diese wesentlich erschien, konnte den Gegenstand eingehender und gewissenhafter Forschung bilden. Es bestand kein Wunsch nach genauerer und tieferer Erkenntnis dessen, was sich außerhalb Europas und andersartig entwickelt hatte. In Europa wie in China gab man sich im 19. Jahrhundert mit ein paar oberflächlichen Kenntnissen vom anderen zufrieden. Das verzerrte und entstellte Bild des anderen schmeichelte der eigenen Eitelkeit und dem Bewußtsein von der eigenen Úberlegenheit.

Aufgrund von Hegels großer Bedeutung für die abendländische Philosophie und für die Erforschung der abendländischen Geschichte haben auch seine Bemerkungen über China stark auf die Nachwelt gewirkt. Die bis heute in konservativen Historikerkreisen - zumal in Deutschland - zuweilen immer noch anzutreffende Meinung, daß Chinas Geschichte für das Abendland unwichtig sei, und daß ein Historiker sich damit nicht zu befassen brauche, geht auf Hegels autoritative Äußerung zurück. Und schließlich entspringen wohl auch die marxistischen Bemühungen, Chinas Geschichte in das von Marx und Lenin aufgrund der abendländischen Entwicklung festgelegte System einer zwangsläufigen historischen Periodisierung zu fassen, der durch Hegel eingeleiteten, so verhängnisvollen Tradition von der weltweiten absoluten Gültigkeit abendländischer Maßstäbe.

8 Wolfgang Franke, China und das Abendland, Göttingen 1962, S. 82.

9 G. W. F. Hegel, Sämtliche Werke, Bd. IX, Philosophie der Weltgeschichte, Hrsg. Georg Lasson, Leipzig

1923, S. 300.

10 Ebd. S. 307.

11 Ebd. S. 311. 


\section{Nachwort des Herausgebers}

Aus Anlaß des Hegeljahres habe ich Herrn Prof. Dr. Wolfgang Franke, Hamburg, gebeten, einen Beitrag über das China-Bild dieses Philosophen zu liefern. Man darf annehmen, daß es Hegel nicht auf China als solches ankommt und daß daher seine zeitbedingten Vorstellungen und Wertungen nicht allzu schwer zu nehmen sind. Ihm ist Geschichte eben nicht, nur irgendwelches Geschehen („ungeschichtliche Geschichte" - Lasson, S. 235), sondern ein teleologischer Prozeß, den das moderne Europa vollzieht, um ihn zu vollenden: „denn Europa ist schlechthin das Ende der Weltgeschichte“ (232). Es liegt von einer solchen Philosophie aus schon um des Zweckes der Darstellung willen nahe, einer solchen eminenten Geschichtlichkeit einen Hintergrund von absoluter Geschichtslosigkeit zu geben. Offenbar hat sich hierfür China als geeignetes Opfer angeboten, weil die Meinung der Zeit in den Chinesen das Volk ohne Geschichte sah (dieselbe Meinung hegt Hegel von Afrika: ein Weltteil, der eigentlich keine Geschichte hat - S. 224). Es steht hier nicht zur Diskussion, wie eine solche Prognose mit der Modernisierung dieser Kontinente $\mathrm{zu}$ vereinbaren ist. Es kommt lediglich darauf an, daß Hegel in seiner Zeit nicht alleingestanden hat, als er China als ein Land der Unveränderlichkeit im allgemeinen, von Geschichtslosigkeit im besonderen vorstellte. Raymond Dawson berichtet ${ }^{1}$ unter der bezeichnenden Uberschrift „» A People of Eternal Standstill«: China and the Historians" über diese Auffassung und ihre Verbreitung bis in die Neuzeit hinein. Danach befindet sich Hegel in guter Gesellschaft: Montesquieu, Condorcet, Herder, Ranke, J. St. Mill ${ }^{2}$ haben dem gleichen Irrtum gehuldigt. Hierher gehört auch A. de Tocqueville. Im Zusammenhang mit dem Nachweis der belebenden Kraft von Wissenschaft führt er als Gegenbeispiel China an ${ }^{3}$. Zwar haben dort die ersten Europäer einen unerwarteten Hochstand von Kunst und Wissenschaft vorgefunden. Aber: „La nation était industrielle; la plupart des méthodes scientifiques s'étaient conservées dans son sein; mais la science elle-même n'y existait plus. Cela leur expliqua l'espèce d'immobilité singulière dans laquelle ils avaient trouvé l'esprit de ce peuple. Les Chinois, en suivant la trace de leurs pères, avaient oublié les raisons qui avaient dirigé ceux-ci. Ils se servaient encore de la formule sans en rechercher le sens; ils gardaient l'instrument et ne possédaient plus l'art de le modifier et de le reproduire. Les Chinois ne pouvaient donc rien changer . ...". Später warnt ${ }^{4}$ er vor einem Krieg der Engländer mit den Chinesen: „Voilà donc enfin la mobilité de l'Europe aux prises avec l'immobilité chinoise!“ - eine Begegnung, in deren Folge chinesische Unbeweglichkeit europäische Beweglichkeit überwältigen wird. Heute glaubt man nicht nur Nachwirkungen dieser Auffassung bemerken zu können ${ }^{5}$-, kein geringerer als Etienne Balasz ${ }^{6}$ vertritt sogar die

1 The Chinese Chameleon - An Analysis of European Conceptions of Chinese Civilization (Oxford UP 1967), S. $64 \mathrm{ff}$.

2 Hierzu auch John M. Robson, The Improvement of Mankind - The Social and Political Thought of John Stuart Mill (Toronto UP 1968), S. $111 \mathrm{f}$.

3 De la Démocratie en Amérique II (Paris 1951), S. 52.

4 Brief an Reeve vom 12. April 1840, in: Correspondance Anglaise, Band VI der Oeuvres Complètes (Paris 1954), S. 58.

5 Vgl. etwa Benjamin I. Schwartz, Communism and China: Ideology in Flux (Harvard UP 1968 ), S. 229.

6 La Bureaucratie céleste (Paris 1968), S. 35. 
Auffassung, daß Hegel im Grunde recht gehabt habe: Zwar entdeckt man heute, wie sehr viele Veränderungen in China im Laufe der Jahrtausende vor sich gegangen sind, aber „il avait raison d'être frappé par le caractère permanent de la société chinoise“. Wie aktuell solche Überlegungen etwa für die Frage des „Ob“ einer modernen chinesischen Außenpolitik sind, bedarf keiner Hervorhebung?.

7 Zwei Bemerkungen seien nachgetragen:

Zum Thema des Beitrages ist zu vergleichen der Vortrag, den Joachim Schickel über „Hegél und China“ auf dem Hegel-Jubiläumskongreß in Stuttgart im Juli 1970 gehalten hat.

Was Afrika angeht, so gibt es heute noch einen Historiker, den Regius Professor der Geschichte an der

Universität Oxford Trevor-Roper, der an der Hegelschen These von der Geschichtslosigkeit auch dieses Erdteils festhält; vgl. hierzu den von J. D. Fage herausgegebenen Sammelband "Africa Discovers Her Past“ (London - Oxford UP 1970), wo diese These mehrfach entschieden verurteilt wird (S. 1, 7, 83). 